\title{
A DINÂMICA DO SISTEMA INOVATIVO DA SAÚDE NO RIO GRANDE DO SUL: UMA ANÁLISE A PARTIR DAS INTERAÇÕES ENTRE OS AGENTES
}

\author{
Ana Lúcia Tatsch \\ Professora do PPGE da Universidade Federal do Rio Grande do Sul (UFRGS) \\ Janaina Ruffoni \\ Professora do PPGE da Universidade do Vale do Rio dos Sinos (UNISINOS) \\ Marisa dos Reis A. Botelho \\ Professora do PPGE da Universidade Federal de Uberlândia (UFU)
}

A concepção de sistemas de inovação permite uma análise do processo inovativo em diferentes âmbitos (nacional, regional/local e setorial). Essa abordagem sistêmica é a que orienta o presente trabalho que visa contribuir para descortinar a dimensão territorial do sistema produtivo e inovativo da saúde no Rio Grande do Sul. A análise de como os diferentes agentes - empresas industriais, hospitais de ensino e grupos de pesquisa - interagem constitui o principal objeto de estudo. A questão analítica central é o exame das fontes de conhecimento para inovar utilizadas pelas empresas industriais e pelos hospitais. O estudo foi realizado através da pesquisa de dados secundários e primários. A investigação de campo deuse com base na aplicação de questionários junto aos atores-chave do sistema em foco. Ao analisar as fontes de conhecimento para a inovação a partir de diferentes perspectivas - grupos de pesquisa, prestadores de serviços (hospitais) e indústria - pontos fortes e fracos do sistema regional de inovação foram revelados. Conclui-se que, além de aumentar a produção científica, é importante que os segmentos produtivos melhor apropriem-se do conhecimento gerado no setor científico.

Palavras-Chave: sistema de inovação em saúde; fontes de conhecimento; interações entre agentes; Rio Grande do Sul.

The concept of innovation systems enables an analysis of the innovation process in different contexts (national, regional/local and sectoral). This is a systemic approach that guides this study helping to uncover the territorial dimension of the production system and innovative health in Rio Grande do Sul. The analysis of how the different agents - industrial firms, teaching hospitals and research groups interact is the main object of study. The central issue is the analytical examination of the sources of knowledge to innovat, generation and diffusion of knowledge and its impact on the provision of healthcare services and products in the territory. The study was conducted through a literature review and research of secondary and primary data. The field research was based on questionnaires along with key actors in the system focused. When analyzing the sources of knowledge for innovation from different perspectives, the research groups, service providers (hospitals) and the industry, strengths and weaknesses of the regional innovation system have been revealed. We conclude that, in addition to increasing the scientific production, it is important to improve the productive sector's use of the knowledge generated in the scientific sector.

Key-words: Health innovation systems; knowledge sources; interactions among agents; Rio Grande do Sul.

JEL: D83, O30 


\section{Introdução}

O estudo da dinâmica do setor da saúde constitui um grande desafio tanto acadêmico quanto políticonormativo. Isso deve-se à complexidade das atividades que envolve - relacionadas à base produtiva de bens de alta intensidade tecnológica e à ampla gama de serviços ofertados (GADELHA, 2003, 2009, 2012) - e à sua importância no âmbito de um projeto de desenvolvimento nacional, dadas suas dimensões, não só social, como também econômica e tecnológica. Tal setor alavanca mais de 8\% do PIB brasileiro e articula a geração e difusão de tecnologias de futuro (FUNDAÇÃO OSWALDO CRUZ et al., 2012).

Face a essas peculiaridades, o sistema produtivo no campo da saúde, cujas atividades econômicas apresentam estreitas relações de interdependência, deve ser entendido a partir de uma dinâmica sistêmica que vai muito além das relações de compra e venda na cadeia produtiva, já que envolve fortes interações e sinergias na geração e difusão de conhecimento, nas relações políticas e institucionais e na formulação de estratégias competitivas (GADELHA, 2009, p.13).

Esta abordagem sistêmica da base produtiva e de inovação em saúde vem sendo trabalhada do ponto de vista acadêmico - Gelijns e Rosemberg, 1995; Albuquerque e Cassiolato, 2000; Gadelha, 2003, 2009 e 2012 - e tem se desdobrado na experiência concreta de política de desenvolvimento no Brasil, no campo industrial e produtivo.

Tal abordagem fundamenta-se na concepção de sistemas de inovação que permite uma análise do processo inovativo em diferentes âmbitos (nacional, regional/local e setorial). Trata-se de uma construção teórica que tem se destacado na literatura evolucionária e neo-shumpeteriana nos estudos de Lundvall (1992), Lundvall et al. (2002), Nelson (1993), Freeman (1995), Johnson (1997), Edquist (1997), Breschi e Malerba (1997) e Cooke (1998).

O Sistema de Inovação compreende um arranjo institucional que envolve uma série de participantes (empresas e suas redes de cooperação e interação, governos, instituições de ensino e pesquisa, de financiamento, entre outros) que interagem e articulam-se entre si. As ligações entre as diversas instituições e suas estruturas de incentivos e capacitações são foco da análise a partir desse referencial. Nesse contexto, a inovação é vista como um processo social e sistêmico.

Essa abordagem sistêmica é a que orienta o presente trabalho que visa contribuir para descortinar a dimensão territorial do sistema produtivo e inovativo de saúde no Brasil, ao examinar tal sistema em um espaço territorial específico, o do Rio Grande do Sul (RS) e, mais particularmente, o de Porto Alegre. Com relação à delimitação territorial, a justificativa para focar Porto Alegre é que boa parte dos serviços de saúde do estado do RS, assim como dos estabelecimentos industriais gaúchos de base química e biotecnológica e de base física, mecânica, eletrônica e de materiais, está concentrada na capital ou na Região Metropolitana de Porto Alegre (RMPA). O mesmo ocorre no caso da estrutura de ensino e pesquisa do estado. Com relação aos grupos de pesquisa com interação, o RS é o segundo estado com maior grupos interativo do país. Soma-se a isso, a relevância da participação do estado nos grupos específicos da área de Ciências da Saúde, já que ocupa também a segunda posição.

O trabalho teve como objetivo geral investigar a dinâmica do sistema de inovação da saúde no Rio Grande do Sul a partir da análise das fontes de conhecimento para inovar utilizadas pelas empresas industriais e pelos hospitais. Valeu-se da abordagem neo-schumpeteriana para analisar as interações entre empresas, universidades e hospitais no sistema inovativo em saúde gaúcho. Considerou-se a relação entre grupos de pesquisa e outras organizações, as relações estabelecidas pelos hospitais universitários e também as relações das empresas que atuam nas indústrias farmoquímicas e de equipamentos médicos.

As perguntas de pesquisa que orientaram a investigação foram as seguintes: Como se dá a dinâmica de inovação no sistema de saúde do RS? Como ocorrem os processos de aprendizagem e que fontes de informação para inovar os agentes do sistema se valem? Com quem os agentes que conformam o sistema interagem visando inovar?

O estudo contou com coleta de dados secundários e primários. Caracteriza-se por ser um estudo empírico de natureza exploratória. 
Além dessa introdução, o texto se organiza em mais três seções. Na segunda, apresentam-se os procedimentos metodológicos; na terceira, os resultados e discussão da pesquisa secundária e de campo; e, na quarta e última, as considerações finais.

\section{Procedimentos Metodológicos}

O presente estudo caracteriza-se por ser um estudo empírico de natureza exploratória que contou com coleta de dados secundários e primários. Buscou-se informações junto a Relação Anual de Informações Sociais (RAIS)/MTE e junto ao Diretório de Grupos de Pesquisa do Conselho Nacional de Desenvolvimento Científico e Tecnológico (DGP / CNPq). Os dados do Censo do Diretório foram analisados a fim de identificar a existência de interações entre grupos de pesquisa e organizações. Para os últimos dados disponíveis, foram construídas redes de interação a partir do programa UCINET.

Os dados primários foram coletados por meio de questionários e entrevistas face-a-face junto aos hospitais universitários e às empresas industriais ${ }^{1}$. Para os atores produtivos optou-se por uma pesquisa por amostragem. Dadas as especificidades e circunstâncias colocadas durante a execução da pesquisa, optou-se por uma amostra não-probabilística, a qual se vale do conhecimento e do julgamento do pesquisador para a seleção dos atores a serem entrevistados, priorizando aqueles que exercem papeis centrais no sistema em foco. O instrumental empregado nas pesquisas de campo consiste de um conjunto de questionários. Os questionários são direcionados aos agentes produtivos - empresas industriais e organizações de serviços.

No caso do RS, os alvos principais da pesquisa empírica foram cinco hospitais de ensino e pesquisa localizados em Porto Alegre; 29 empresas industriais da RMPA ligadas à saúde, sendo 12 de base química e biotecnológica e 17 de base mecânica, eletrônica e de materiais; e 10 líderes ou integrantes de Grupos de Pesquisa do Diretório do CNPq, na sua maioria da área da medicina.

\section{Características do sistema inovativo gaúcho da saúde}

Uma representação do sistema inovativo da saúde pode ser vista na Figura 1. O sistema inovativo da saúde abarca um conjunto de empresas industriais tanto de base química e biotecnológica, quanto de base mecânica, eletrônica e de materiais, além de um conjunto de organizações prestadoras de serviços em saúde que são os principais consumidores dos produtos manufaturados.

O subsistema de serviços, através da assistência médica - envolvendo hospitais, clínicas, postos médicos, entre outros - interage não só com as indústrias do setor, mas também com outros agentes, como as instituições de ensino e pesquisa. O mercado de serviços de saúde é cada vez mais pressionado pelos custos e influenciado por importantes fatores, tais como: mudanças epidemiológicas, demandas demográficas, mudanças político-econômicas e desenvolvimento de novas soluções tecnológicas para os problemas da saúde.

A forma como os serviços de saúde interagem com outros agentes do sistema implica em atribuir a estas organizações um papel chave dentro do sistema, que extrapola em muito o de consumidores passivos dos produtos manufaturados (BARBOSA; GADELHA, 2012; DJELALL; GALLOUJ, 2008; WINDRUM; GARCÍA-GOÑI, 2008). Os hospitais que abrigam tratamentos de alta complexidade e/ou atividades de pesquisa clínica são organizações que interagem ativamente com as instituições de pesquisa científica e com a indústria química, farmacológica e a de base metal-mecânica. Mais recentemente, pode-se destacar também a forte interação com as tecnologias de informação e comunicação (TICs), dada a sua forte presença na gestão dos serviços de saúde e na facilitação do acesso a serviços qualificados, como é o caso da telemedicina.

\footnotetext{
${ }^{1}$ Esta coleta empírica foi desenvolvida no âmbito do Projeto de Pesquisa "Territorialização do Complexo EconômicoIndustrial da Saúde: a evolução e dinâmica de Arranjos e Sistemas Produtivos e Inovativos Locais no Brasil” coordenado pela Rede de Pesquisa em Sistemas e Arranjos Produtivos e Inovativos Locais (RedeSist IE/UFRJ) e pela Fundação Oswaldo Cruz (Fiocruz), com financiamento do Centro de Gestão e Estudos Estratégicos (CGEE).
} 


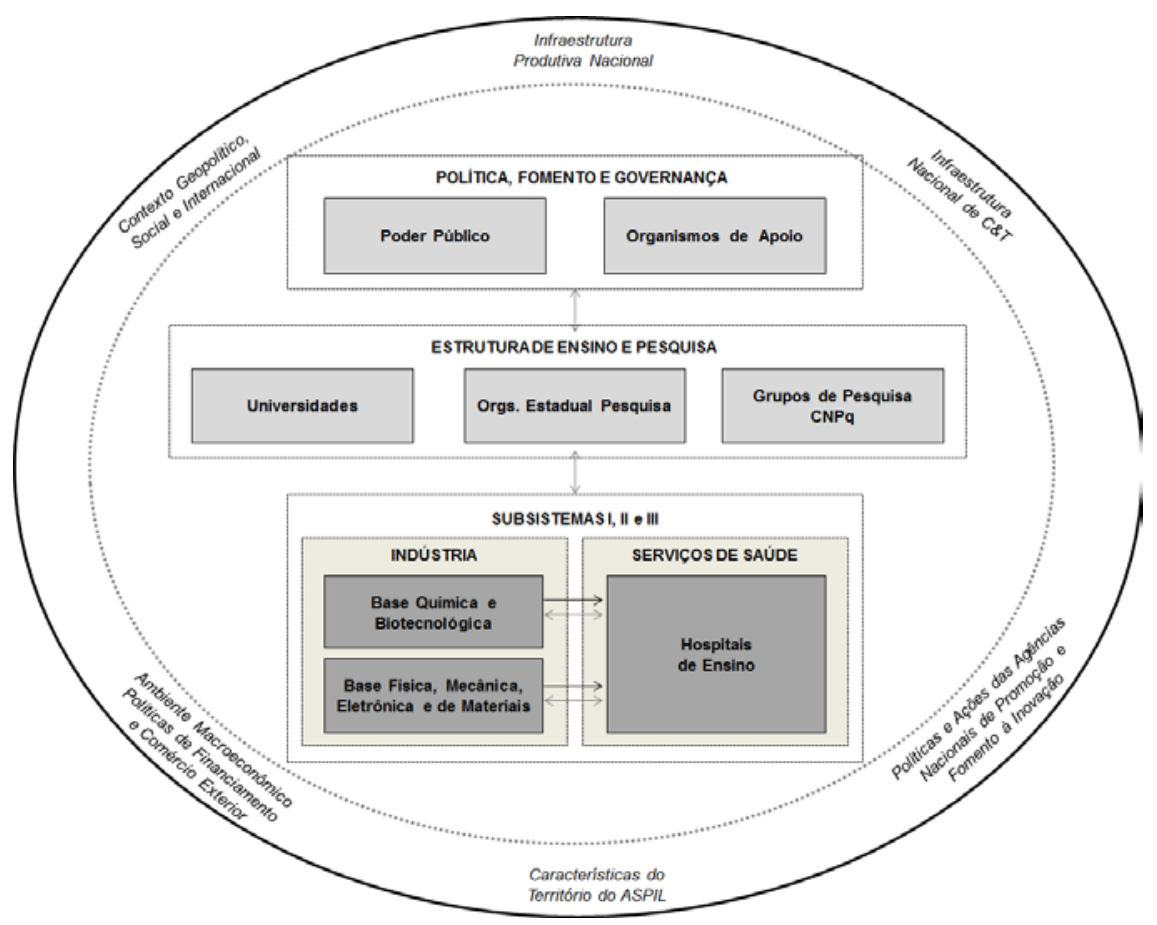

Figura 1 - Sistema de Inovação em Saúde

Fonte: Tatsch; Batisti; Fraga (2013).

As universidades e instituições de pesquisa têm também um importante lugar nesse sistema, especialmente pela proximidade que o progresso tecnológico do setor tem com a ciência, oportunizando que se avance na oferta de bens e serviços qualificados.

Também o Estado cumpre um importante papel na dinâmica do sistema de saúde, através de ações, explícitas ou implícitas, e adquire uma abrangência dificilmente encontrada em outro sistema produtivo. Estas ações abrangem (i) compra de bens e serviços; (ii) repasses de recursos para os prestadores de serviços; (iii) investimentos na indústria e na rede assistencial; além de (iv) um conjunto amplo de atividades regulatórias que delimitam as estratégias dos agentes econômicos. O Estado constitui, assim, uma instância determinante da dinâmica industrial do sistema, graças ao seu elevado poder de compra de bens e serviços e de indução e às atividades regulatórias que desempenha, numa forte interação com a sociedade civil organizada.

A relevância atribuída à prestação de serviços de saúde e às universidades e instituições de pesquisa dentro desta abordagem teórica implica considerar o território em que estas atividades são desenvolvidas. Os impactos oriundos das atividades, tanto dos prestadores de serviços de saúde como dos relacionados à pesquisa e desenvolvimento tecnológico, apresentam-se de forma importante nos territórios em que estão localizadas (COOKE, 1998; ASHEIM; GERTLER, 2006; CASSIOLATO; LASTRES, 1999).

A partir dos dados da RAIS, observa-se que, embora RS represente o quarto estado em ordem de importância na estrutura industrial brasileira focada em saúde, sua participação em nível nacional é pequena. Quanto ao número de estabelecimentos e empregos por segment no RS, como mostrado nas Tabelas 1 e 2, o número de empresas de base mecânica é mais significativo vis-à-vis aquele das de base química e biotecnológica. O mesmo ocorre quanto aos postos de trabalho gerados. 
Table 1- Estabelecimentos das Indústrias de Base Química e Biotecnológica e de Base Física, Mecânica, Eletrônica e de Materiais no RS (2014)

\begin{tabular}{|c|c|c|c|}
\hline \multirow{2}{*}{ Segmentos Industriais } & \multicolumn{3}{|c|}{ RS } \\
\hline & $\mathbf{N}^{\circ}$ & \% Total RS & \% Brasil \\
\hline Fabricação de Produtos Farmoquímicos & 11 & $0,18 \%$ & $7,24 \%$ \\
\hline Fabricação de Medicamentos para Uso Humano & 28 & $0,46 \%$ & $5,91 \%$ \\
\hline Fabricação de Preparações Farmacêuticas & 2 & $0,03 \%$ & $4,65 \%$ \\
\hline Total & 41 & $0,67 \%$ & $6,13 \%$ \\
\hline $\begin{array}{l}\text { Fabricação de Aparelhos Eletromédicos e Eletroterapêuticos e Equipamentos de } \\
\text { Irradiação }\end{array}$ & 8 & $0,38 \%$ & $3,16 \%$ \\
\hline $\begin{array}{l}\text { Fabricação de Instrumentos e Materiais para Uso Médico e Odontológico e de } \\
\text { Artigos Ópticos }\end{array}$ & 301 & $14,32 \%$ & $7,46 \%$ \\
\hline Total & 309 & $14,70 \%$ & $7,20 \%$ \\
\hline
\end{tabular}

Fonte dos dados brutos:RAIS / MTE (2014).

Tabela 2 - Empregos das Indústrias de Base Química e Biotecnológica e de Base Física, Mecânica, Eletrônica e de Materiais no RS (2014)

\begin{tabular}{|c|c|c|c|}
\hline \multirow{2}{*}{ Segmentos Industriais } & \multicolumn{3}{|c|}{ RS } \\
\hline & $\mathbf{N}^{\circ}$ & \% Total RS & \% Brasil \\
\hline Fabricação de Produtos Farmoquímicos & 299 & $0,73 \%$ & $5,72 \%$ \\
\hline Fabricação de Medicamentos para Uso Humano & 1.673 & $4,06 \%$ & $1,92 \%$ \\
\hline Fabricação de Preparações Farmacêuticas & 52 & $0,13 \%$ & $3,87 \%$ \\
\hline Total & 2.024 & $4,91 \%$ & $2,16 \%$ \\
\hline $\begin{array}{l}\text { Fabricação de Aparelhos Eletromédicos e Eletroterapêuticos e Equipamentos de } \\
\text { Irradiaçâ }\end{array}$ & 135 & $1,29 \%$ & $2,42 \%$ \\
\hline $\begin{array}{l}\text { Fabricação de Instrumentos e Materiais para Uso Médico e Odontológico e de } \\
\text { Artigos Ópticos }\end{array}$ & 3.210 & $30,56 \%$ & $5,45 \%$ \\
\hline Total & 3.345 & $31,85 \%$ & $5,19 \%$ \\
\hline
\end{tabular}

Fonte dos dados brutos: RAIS / MTE (2014).

Com relação à infraestrutura de ensino e pesquisa, no RS, 58 instituições de ensino superior ofertam 413 cursos de graduação na área da saúde, disponibilizando mais de 33 mil vagas para formação de nível superior (e-MEC / MEC, 2012). No que tange à formação em nível de pós-graduação strictu sensu, existem no estado 70 cursos - 41 de mestrado (acadêmico e profissional) e 29 de doutorado ofertados por 12 instituições de ensino (CAPES, 2012). Em Porto Alegre, quatro universidades Universidade Federal do Rio Grande do Sul, Fundação Universidade Federal de Ciências da Saúde de Porto Alegre, Pontifícia Universidade Católica do Rio Grande do Sul e Fundação Universitária de Cardiologia - ofertam 14 cursos de medicina em nível de pós-graduação strictu sensu, com 50 áreas de concentração. São justamente tais universidades que se valem dos hospitais ora examinados enquanto hospitais-escola.

Há também um histórico em pesquisa bastante importante no estado. Na seção seguinte, procurase justamente melhor compreender com quais agentes os grupos de pesquisa interagem e que tipo de interações são mais frequentes.

\subsection{Interações entre grupos de pesquisa e organizações}

Em 2010, o número de grupos de pesquisa com interação cadastrados no DGP, considerando todas as áreas de conhecimento, era de 404 no RS, sendo este o segundo estado do país em termos de grupos com interação.

Na área de 'Ciências da Saúde', entre os cinco principais estados com maior número de grupos de pesquisa com interação em 2002 e 2010 do país, o RS estava na segunda posição, com um crescimento de 16 grupos com interação em 2002 para 53 em 2010. Também é importante apontar que comparando a 
área de Ciências da Saúde com outras áreas de conhecimento no RS, essa área alcança a terceira posição em número de grupos com interação, ficando atrás das áreas de Engenharias e Ciências Agrárias.

A Tabela 3 mostra o total de grupos de pesquisa com interação, o número de interações e de organizações com as quais os grupos interagem.

Tabela 3 - Número de grupos de pesquisa com interação, de interações e de organizações na área da saúde no BR e no RS (2010)

\begin{tabular}{l|c|c|c|c|c|c|c|c|c|}
\hline \multirow{2}{*}{ Área } & $\begin{array}{c}\text { Grupos de Pesquisa } \\
\text { com interação }\end{array}$ & \multicolumn{2}{|c|}{ Número de interações } & \multicolumn{3}{|c|}{$\begin{array}{c}\text { Número de } \\
\text { Organizações }\end{array}$} \\
& RS & BR & (\%)RS/BR & RS & BR & (\%)RS/BR & RS & BR & (\%)RS/BR \\
\hline Educação Física & 8 & 32 & 25.0 & 71 & 123 & 57.7 & 43 & 71 & 63.4 \\
Enfermagem & 6 & 45 & 13.3 & 8 & 119 & 6.7 & 4 & 55 & 7.3 \\
Farmácia & 5 & 55 & 9.1 & 28 & 207 & 13.5 & 14 & 101 & 14.9 \\
Fisioterapia e & & & & & & & & & \\
Terapia & & & & & & & & & \\
Ocupacional & 3 & 19 & 15.8 & 6 & 36 & 16.7 & 6 & 27 & 22.2 \\
Fonoaudiologia & 0 & 8 & 0.0 & 0 & 30 & 0.0 & 0 & 14 & 0.0 \\
Medicina & 19 & 132 & 14.4 & 40 & 352 & 11.4 & 21 & 163 & 14.1 \\
Nutrição & 1 & 17 & 5.9 & 3 & 34 & 8.8 & 3 & 25 & 12.0 \\
Odontologia & 5 & 59 & 8.5 & 16 & 218 & 7.3 & 8 & 75 & 10.7 \\
Saúde Pública & 6 & 63 & 9.5 & 19 & 172 & 11.0 & 13 & 106 & 13.2 \\
\hline Total & $\mathbf{5 3}$ & $\mathbf{4 3 0}$ & $\mathbf{1 2 . 3}$ & $\mathbf{1 9 1}$ & $\mathbf{1 2 9 1}$ & $\mathbf{1 4 . 8}$ & $\mathbf{1 1 2}$ & $\mathbf{6 3 7}$ & $\mathbf{1 8 . 5}$ \\
\hline
\end{tabular}

Fonte: Diretório de Grupos de Pesquisa (DGP) - CNPq, Censo, 2010.

É possível analisar na Tabela 3 que em algumas áreas do conhecimento o RS tem uma densidade do número de interações por grupo de pesquisa elevada, como em educação física, fisioterapia e farmácia. Considerando a densidade em termos de organizações com as quais os grupos interagem, as maiores densidades estão nas mesmas áreas citadas anteriormente: educação física, fisioterapia e farmácia.

A partir da análise das interações entre grupos de pesquisa e um conjunto diversificado de organizações - como, por exemplo, hospitais e empresas - foram construídas duas redes que representam as interações, permitindo uma melhor compreensão de suas estruturas, atores e áreas de conhecimento que se destacam. Como identificado, em 2010 havia um total de 53 grupos de pesquisa de Ciências da Saúde no RS que interagiram com 112 organizações diferentes.

Antes de observar a Figura 2, é importante notar que: (1) todas as áreas do conhecimento da Ciências da Saúde estão representadas na rede e receberam as seguintes siglas: Educação Física (ef); Enfermagem (en); Farmácia (far); Fisioterapia e Terapia Ocupacional (fto); Fonoaudiologia (fono); Medicina (med); Nutrição (nut); Odontologia (odo); e Saúde Pública (sc); e (2) as organizações foram classificadas como: Entidades de Negócios (EE), Entidades Sem Fins Lucrativos (ESL) e da Administração Pública (AP). A rede é organizada por instituições, especialmente as universidades, mas também institutos de pesquisa e hospitais. Esses atores são representados por nódulos redondos e são identificados pela instituição e área de especialização. Assim, o símbolo 'IBTEC Ef.1', significa o grupo de pesquisa 1 na área de Educação Física (ef) do Instituto Brasileiro de Tecnologia do Couro, Calçados e Artefatos (IBTEC). Observa-se que vários destes nódulos são universidades que têm interação com grupos, especialmente UFRGS e PUCRS. Além destes, também se destacam outros atores: o IBTEC, o Hospital de Clínicas de Porto Alegre (HCPA) e do Instituto de Cardiologia (IC-FUC).

No que diz respeito às organizações, observa-se que a maior parte é Entidade de Negócios (EE). O número ao lado de cada sigla foi usado para identificar os atores, quantificá-los e verificar a quantidade de interação para cada ator. Observou-se mais de 80 Entidades de Negócios (EE) na rede, o qual é o principal grupo de entidades com as quais os grupos interagem; os demais são Entidades Sem Fins Lucrativos (ESL) e Administração Pública (AP). A maioria das EE foi citada principalmente por um ou 
dois grupos de pesquisa, como pode ser visto na Figura 2 pelo número de linhas com setas que apontam para os nódulos de várias formas (triângulos, diamantes, quadrados). Vale ressaltar aqui que o EE4 foi citado por oito grupos de pesquisa alocados na UFRGS e HCPA. A maioria das entidades empresariais (EE) são de tamanho médio e grande. Isto pode ser visto pela forma dos nódulos identificados como EE: grandes empresas estão no formato "quadrado", médias no formato "diamante" e as pequenas e micro estão no formato "triângulo".

Também é possível observar a questão da proximidade geográfica nas interações estabelecidas. As linhas sólidas de ligação entre os nós representam a interação com as entidades do setor produtivo, localizados no RS e as linhas pontilhadas refletem interações com entidades de outros estados do Brasil. Observa-se na Figura 2, uma predominância (56\%) das interações entre os grupos e organizações localizadas em RS. Isso corrobora as conclusões gerais da literatura (COOKE, 1998; ASHEIM; GERTLER 2006) e os achados de Costa, Ruffoni e Puffal (2011), que identificaram a proximidade geográfica como uma característica da interação universidade-empresa no RS.

A este respeito, é importante observar o comportamento de dois grupos de pesquisa na área de Farmácia (far) e Odontologia (odo) da Universidade Federal de Santa Maria (UFSM). Na rede apresentada, é possível observar dois nós, 'UFSM.far 1' e 'UFSM.odo 1', que relatam ter interações unicamente com organizações localizadas fora do RS.

Finalmente, é importante mencionar que alguns atores da rede concentram grande parte das interações: o IBTEC com um grupo de pesquisa único na área de Educação Física, e a UFRGS, com diversos grupos de pesquisa em diferentes áreas do conhecimento. Os papéis desempenhados por esses atores são: 1) IBTEC é uma instituição de geração de conhecimento tecnológico centrado principalmente na área de Educação Física com pontos de conexão com várias empresas do setor calçadista; e 2) a UFRGS é uma instituição de ensino pública, centenária, reconhecida nacional e internacionalmente. Está localizada em Porto Alegre e possui grupos de diferentes áreas do conhecimento, e, portanto, tem um papel mais central no conjunto de grupos de pesquisa com interação na área de Ciências da Saúde no estado. 


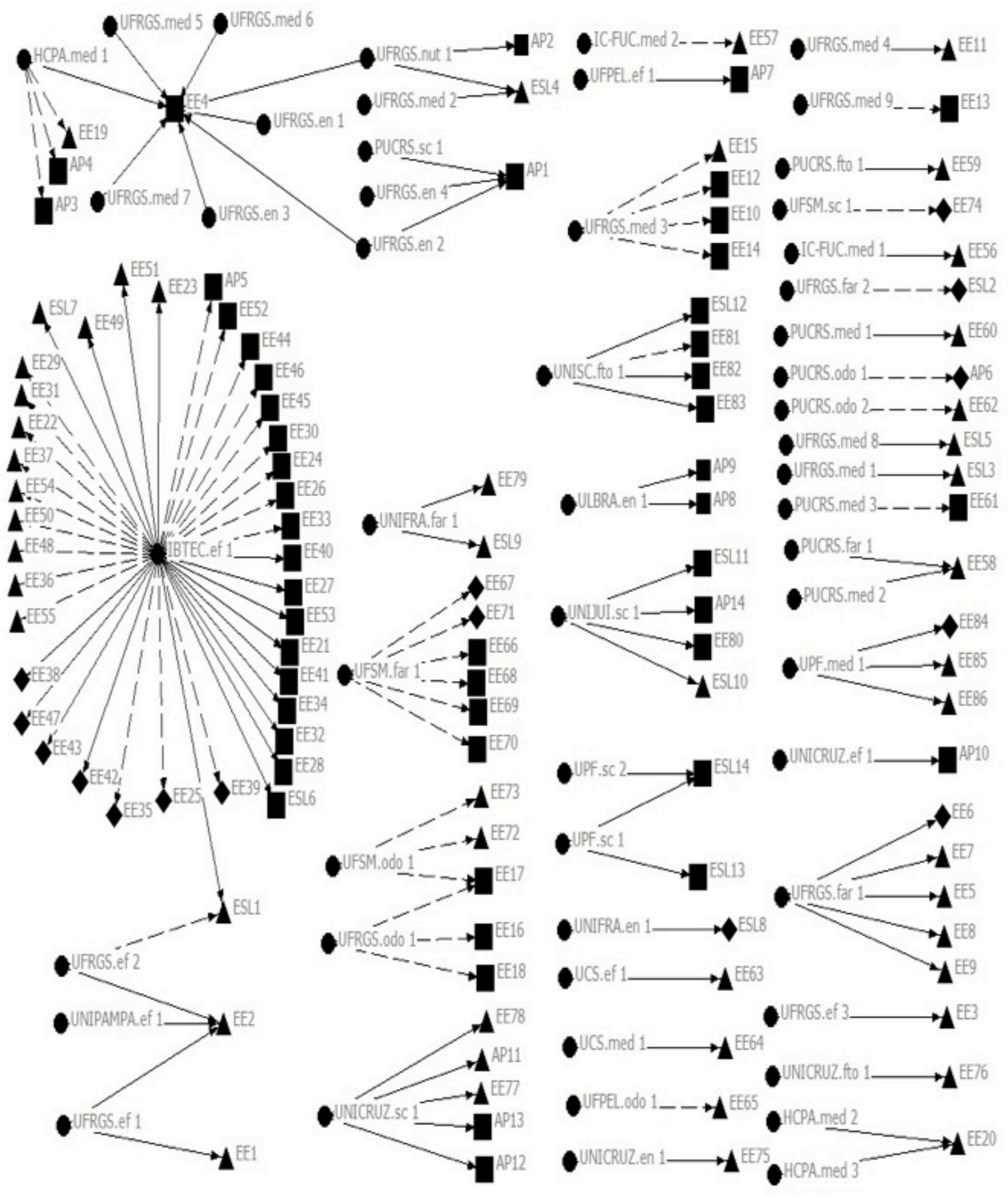

Figura 2 - Atores e redes estabelecidas na área das Ciências da Saúde no RS (2010) Source: DGP, Censo de 2010.

As organizações do setor produtivo que estão na rede foram classificadas conforme a Classificação Nacional de Atividades Econômicas (CNAE). A Tabela 4 apresenta a classificação CNAE das organizações que interagem com os grupos de pesquisa na área de Ciências da Saúde no RS. 
Tabela 4 - Classificação das organizações que interagem com os grupos de pesquisa, conforme a CNAE, RS (2010)

\begin{tabular}{llc}
\hline \multicolumn{1}{c}{ Classificação Nacional das Atividades Econômicas (CNAE) } & Número de Organizações \\
\hline 1. & Fabricação de calçados & 25 \\
2. & Fabricação de produtos farmacêuticos & 17 \\
3. Atividades de atendimento hospitalar & 13 \\
4. Administração do estado e da política econômica e social & 12 \\
5. Atividades de serviços de complementação diagnóstica e terapêutica & 5 \\
Outros & 43 \\
\hline
\end{tabular}

Fonte: Diretório de Grupos de Pesquisa do CNPq (DGP - CNPq), Censo de 2010.

A primeira atividade em termos de número de organizações é ‘Fabricação de Calçados’. Os outros setores que se destacam são: 'fabricação de produtos farmacêuticos’ (com 17 organizações na rede), 'Atividades de atendimento hospitalar' (13 organizações) e 'Administração do estado e da política econômica e social’ (12 organizações).

Para observar a participação das diferentes áreas do conhecimento nas interações estabelecidas pelos grupos de pesquisa investigados, foi construída a Figura 3. É importante mencionar que os nódulos redondos representam as diferentes áreas do conhecimento e o número de grupos que o compõem. Assim, por exemplo, 'med.19' é a área da medicina com 19 grupos, independentemente da instituição de pesquisa a que pertencem os grupos. Todas as demais configurações de rede permanecem as mesmas da Figura 2 e foram explicitadas anteriormente.

Observa-se na Figura 3 que somente a área de Fonoaudiologia (fono. 0) não tem grupo de pesquisa com interação. As maiores concentrações de grupos de pesquisa com interação estão nas áreas de Medicina e Educação Física. Dos 53 grupos, 19 estão em Medicina, o que representa 36\% do total. A área de Educação Física (ef. 8) tem 8 grupos. A área de conhecimento de Saúde Pública (sc. 6) tem o terceiro maior contingente de grupos interativos, juntamente com a área de Enfermagem (en. 6). Os grupos da Farmácia (far. 5) interagem com 15 organizações. As instituições parceiras estão localizadas predominantemente no RS e são, em grande parte, de micro e pequeno porte.

A área de Odontologia (odo. 5) tem cinco grupos de pesquisa, e estas interagem com 8 organizações, e apenas uma delas é caracterizada como Administração Pública (AP), as demais são Entidades de Negócios (EE). É importante atentar para o fato de que nenhuma das 8 organizações está localizada no RS e tal fenômeno foi observado neste estudo somente para essa área do conhecimento. 


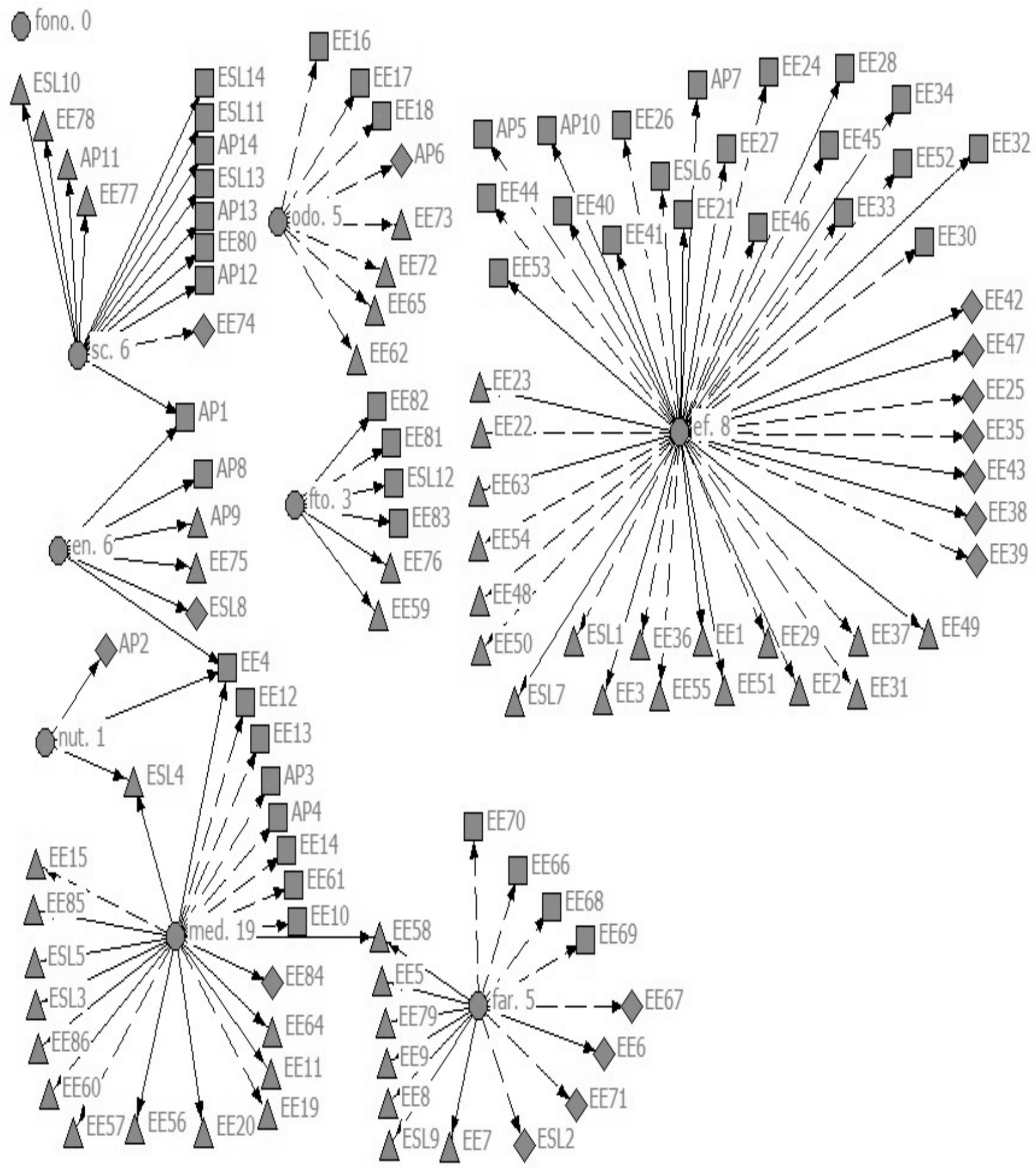

Figure 3 - Áreas de conhecimento dos grupos interativos da área de Ciências da Saúde do RS, 2010. Fonte: Diretório de Grupos de Pesquisa (DGP - CNPq), Censo de 2010.

Em suma, destaca-se que:

- A área de Educação Física tem uma alta concentração das interações, as quais são realizadas pelo IBTEC. Ao contrário de outras instituições identificadas neste estudo, o IBTEC é uma instituição ligada à indústria do calçado, que é caracterizada por ser uma das principais especializações produtivas da indústria do RS. Tal fenômeno explicita a existência de uma conexão entre a necessidade tecnológica de um importante setor produtivo e a infraestrutura de geração de conhecimento tecnológico local. 
- Em termos de atores, verifica-se o destaque da UFRGS. Esta universidade tem 20 grupos de pesquisa, que representam 38\% de todos os grupos de pesquisa com interação na área das Ciências da Saúde no RS. Tais grupos interagem com 25 organizações de diferentes tipos (17 empresas, 5 organizações sem fins lucrativos, 2 entidades classificadas como administração pública e 1 entidade hospitalar). Além disso, os grupos interativos da UFRGS estão distribuídos em diferentes áreas do conhecimento, com destaque para a Medicina;

- A 'proximidade geográfica' mostra-se como um fator importante nas interações, pois identificou-se que $56 \%$ de todas as organizações com as quais os grupos interagem estão localizadas no RS; e

- As organizações de grande porte são prevalentes no grupo.

\subsection{Resultados da pesquisa de campo}

A fim de investigar a estrutura de serviços do sistema local de inovação em saúde na RMPA, foram examinados empiricamente cinco hospitais de ensino e pesquisa: Grupo Hospitalar Conceição (GHC), Hospital de Clínicas de Porto Alegre (HCPA), Hospital São Lucas da PUCRS (São Lucas PUCRS), Instituto de Cardiologia (IC) e Irmandade Santa Casa de Misericórdia de Porto Alegre (Santa Casa). No conjunto, esses hospitais oferecem 4.525 leitos (Especializado Cirúrgico, Especializado Clínico, Obstétrico, Pediátrico, Hospital Dia, UTI, e outras especialidades). Dentre os hospitais analisados, é o GHC o que oferece o maior número de leitos, 1.658. Já a Santa Casa é aquela que possui a maior infraestrutura de centros cirúrgicos e ambulatórios. No geral, o IC é a instituição que apresenta menor infraestrutura. Isso se deve à sua especialização na cardiologia.

Levando em conta essas informações, destaca-se a relevância desses hospitais, considerados de alta complexidade, no contexto de atendimentos realizados no estado. Têm importante papel na assistência não só da população da capital, mas de todo o RS.

Dentre os hospitais analisados, o GHC é o que atende exclusivamente ao Sistema Único de Saúde (SUS) e constitui-se na maior rede pública de hospitais do sul do país. É vinculado ao Ministério da Saúde. Seus quatro hospitais foram responsáveis por 35\% de todas as internações pelo SUS em Porto Alegre em 2009. O Hospital Conceição conta com 5.001 funcionários e é o maior hospital do Grupo (GHC), que possui ao todo três hospitais. Funciona 24 horas por dia e possui todas as especialidades de um hospital geral, através do ambulatório, da emergência e da internação. Vale destacar que o Serviço Saúde Comunitária do GHC foi modelo para o Ministério da Saúde na criação do Programa de Saúde da Família.

O HCPA é uma empresa pública de direito privado, considerado referência nacional de hospital universitário. Conta com patrimônio próprio e autonomia administrativa em relação à Universidade Federal do Rio Grande do Sul (UFRGS), do qual é hospital-escola, sendo subordinado ao Ministério da Educação. Do total da sua capacidade instalada, 85\% são destinados a pacientes do SUS, contando, atualmente, com mais de 5 mil colaboradores.

O HSL, hospital-escola da Pontifícia Universidade Católica do Rio Grande do Sul (PUCRS), entidade jurídica de direito privado, é uma instituição mantida pela Província Marista. Classificado como hospital geral e de natureza filantrópica, atende praticamente todas as especialidades médicas em adultos e pediatria, com alguns centros de excelência como a área de neurologia, cardiologia, cirurgia minimamente invasiva, etc. Dentre os atendimentos em 2011, 61\% foram realizados pelo SUS.

O IC é uma fundação de direito privado sem fins lucrativos. Sua história começa em 1946, como uma unidade ambulatorial. Por ser uma instituição filantrópica, 70\% dos seus atendimentos são pelo SUS. Diversos procedimentos atualmente realizados pala instituição foram pioneiros no RS, na Região Sul, no Brasil e no mundo. O primeiro transplante de coração do RS foi realizado em 1984 por equipe de médicos do IC. Também realizou o primeiro implante de marcapasso no mundo com a utilização do robô Aesop 3000. Os primeiros procedimentos do Sul do Brasil de implante de válvula aórtica pela via percutânea e as aplicações pioneiras da terapia gênica para tratar cardiopatia isquêmica terminal através do uso de plasmídeo representam ainda este pioneirismo. 
A Santa Casa é uma instituição privada de caráter filantrópico criada em 1803. O denominado Complexo Santa Casa é constituído por sete hospitais, sendo dois hospitais gerais (um para atendimento de adultos e o outro de crianças) e cinco especializados em cardiologia, neurocirurgia, pneumologia, oncologia (segundo maior prestador de radioterapia para o SUS no país) e transplantes. Da totalidade dos atendimentos, $60 \%$ dos procedimentos realizados no Complexo são em pacientes advindos do SUS representatividade que coloca a Santa Casa como a instituição privada que mais presta serviços ao SUS no RS.

No que diz respeito à gestão desses hospitais, independentemente se públicos ou filantrópicos, há um nítido processo de profissionalização das gestões ao longo dos últimos anos. Vários processos internos dos hospitais, relacionados às suas diversas áreas de operação, foram otimizados e tornados mais eficientes. Em função disso, instituições como a Santa Casa e o HCPA estão preparando-se para buscar certitificação internacional, o acreditamento junto à Joint Commission International.

O Hospital de Clínicas é referência em gestão de Hospitais Universitários (HUs), desempenhando papel relevante no âmbito do Programa Nacional de Recuperação dos Hospitais Universitários (REHUF) do Ministério da Educação. O Clínicas participa das ações deste programa desde 2009, quando foi escolhido pelo MEC para transferir seu modelo de gestão aos demais HUs da rede, com o desenvolvimento do Aplicativo de Gestão dos Hospitais Universitários.

Também o GHC passou por um processo de reorganização organizacional. Houve a incorporação dos seus vários hospitais no Conceição, unificando vários dos seus processos, atinentes às compras e aos recursos humanos, por exemplo. Os processos licitatórios passaram a ser realizados exclusivamente por meio eletrônico.

Ainda quanto a inovações organizacionais, o serviço de higienização têxtil foi terceirizado. Pretende-se ainda desenvolver um projeto de lavanderia conjunta. Isto é, o GHC em conjunto com outros hospitais de Porto Alegre - Mãe de Deus, Ernesto Dorneles, Divina Providência e HCPA - serão parceiros na compra da empresa que presta esse serviço de higienização. Em consequência, haverá uma significativa redução de gastos nessa área. A ideia é ampliar essa sistemática para outros serviços, como a higienização de materiais e a engenharia clínica, que envolve planejamento, compra e testagem de equipamentos. Ações dessa natureza, que envolvem parcerias e cooperação em áreas sensíveis da operação dos hospitais, podem ser melhor exploradas, já que ao serem adotadas oportunizam ganhos a todos os envolvidos.

Enfim, verificou-se que inovações organizacionais e incorporação de sistemas de gestão têm refletivo de forma bastante positiva no modo de operação das organizações investigadas e na oferta de seus serviços.

Além do reconhecimento do esforço na gestão, há ainda o entendimento de que a qualidade dos serviços médicos prestados nesta região, com impactos em todo o estado, é também decorrência da boa formação médica no RS.

A pesquisa encontra espaço privilegiado no HCPA que possui um Centro de Pesquisa Experimental e disponibiliza infraestrutura com seis laboratórios multiusuários e área física para o desenvolvimento de projetos específicos em 20 laboratórios temáticos. O Centro realiza pesquisa experimental básica e aplicada.

No IC, a pesquisa também se divide em básica e aplicada. A primeira volta-se, especialmente, para os trabalhos com células-tronco, e podem ter como objeto a regeneração de miocardio depois do infarte. Já a segunda é voltada para a clínica, com aplicação na cardiologia. Na linha da pesquisa aplicada, várias são as temáticas dos projetos desenvolvidos: arteriosclerose; coração artificial; cardiologia fetal; hemodinâmica focada nareestenosepós-angioplastia; angioplastia primária do infarto agudo; epidemiologia dogaúcho, dentreoutras. O Centro de Pesquisa Clínica atua também na testagem de drogas e órteses.

Quanto à pesquisa no HSL, esse conta com um Centro de Pesquisa Clínica para testagem de medicamentos, com financiamento de indústrias farmacêuticas. Atualmente, 215 pesquisas estão em andamento em diversas áreas com destaque para a oncologia, cardiologia, pneumologia e reumatologia. 
Junto ao hospital ainda funcionam dois institutos de pesquisa da universidade: o Instituto de Geriatria e Gerontologia e o Instituto de Pesquisa Biomédica.

No geral, a partir da investigação junto a esses hospitais, identificou-se que a pesquisa ocorre em grande parte vinculada à testagem de novas drogas. Isto é, há em todos os centros examinados participação em protocolos internacionais de testagem de novas drogas. São estudos multicêntricos, normalmente financiados por empresas multinacionais produtoras de medicamentos. Há consenso entre os entrevistados de que tal envolvimento gera várias vantagens, especialmente aos pacientes. Neste caso, permitem acesso a tratamentos aos pacientes que teriam que entrar em espera no sistema de saúde. Viabilizam não só tratamento, mas acompanhamento a possíveis intercorrências e exames. Muitas vezes incluem pacientes que já passaram por falha de outros tratamentos. Também oportunizam aos médicos, quando as drogas chegam ao mercado, saber manejar com elas. Além disso, têm ainda um impacto positivo sobre a qualidade da pesquisa em decorrência da série de requisitos a serem cumpridos e sobre o reconhecimentos dos nossos cientistas.

No caso da oncologia, por exemplo, comparativamente à cardiologia, há mais testagem de fármacos. Embora na cardio também ocorra, a testagem de procedimentos de cirurgia, avaliação de tipos de cateterismo, de intervenção inter-cutânea, entre outros, são mais freqüentes.

Além das pesquisas dessa natureza, há também aquelas propostas pelos pesquisadores e financiadas pelos órgãos de fomento tradicionais, como CNPq, FINEP e FAPERGS. Como resultado, se tem a geração de conhecimentos divulgados através de publicações em periódicos nacionais e internacionais e, algumas vezes, a geração de patentes. A regra não é a geração de produtos, mas a produção de artigos científicos.

Também há consenso no entendimento de que o envolvimento nessas atividades de pesquisa, de forma geral, tem um impacto positivo na assistência médica e no ensino. Isto é, os conhecimentos gerados são automaticamente incorporados nos procedimentos da assistência médica, permitindo aplicação terapêutica. Logo, inovações incrementais estão continuamente ocorrendo. Da mesma forma, que os conhecimentos gerados são prontamente transmitidos aos alunos/residentes.

Quanto à relação com os pesquisadores docentes, já que os hospitais ora em foco são justamente de ensino e, portanto, possuem proximidade com as universidades que os têm enquanto escola, não há homogeneidade nos hospitais pesquisados. O IC-FUC seria um exemplo de estreita proximidade entre os professores/pesquisadores do PPG em Cardiologia com a assistência médica prestada pelo Instituto. Mas essa não é a regra, muitas vezes o docente pesquisador não tem atuação enquanto médico no hospitalescola e desenvolve suas pesquisas sem vinculação com as atividades de assistência. Assim, embora pareça que por serem hospitais-escola haja uma estreita relação com as universidades parceiras isso não é estabelecido a priori no que tange à pesquisa.

Com relação às transações comerciais dos hospitais - aquisição de medicamentos, fármacos, equipamentos, materiais, serviços de manutenção, entre outros -, essas são realizadas normalmente através de licitações. Em algumas situações, por pregões federais, como no caso do HCPA, ou por licitações realizadas pelo próprio hospital, como no caso do GHC. Em função da utilização deste mecanismo de compra, são os preços o atributo relevante para contratação. Em consequência desse processo, não há nenhuma garantia de que empresas gaúchas sejam fornecedoras e, na realidade, normalmente não o são. Boa parte dos produtos adquiridos tem como origem empresas de fora do estado e, em muitos casos, os produtos vem de fora do Brasil. Naqueles hospitais de natureza privada filantrópica, as compras ocorrem por seleção das propostas dos fornecedores. Mais uma vez são poucas as empresas gaúchas que aparecem nesta lista.

De forma geral, foi destacado que a indústria nacional, com algumas exceções, ainda não é produtora de produtos de maior complexidade, sejam medicamentos sejam equipamentos. No caso dos equipamentos, particularmente daqueles com maior tecnologia embutida, como dos destinados ao diagnóstico e tratamento de câncer - equipamentos de imagem, ressonância, tomografia por emissão de pósitrons, radioterapia, entre outros -, a produção se dá no exterior, concentrada em algumas poucas empresas de grande porte que investem muito em $\mathrm{P} \& \mathrm{D}$. O mesmo ocorre com os medicamentos de ponta 
que são também produzidos por grandes empresas multinacionais. É unânime o entendimento de que o gap tecnológico entre o produto importado e o nacional de boa parte dos produtos da área da saúde é imenso.

Quanto às possíveis interações entre os serviços em saúde e a base produtiva estadual, do ponto de vista das empresas industriais gaúchas também alvo da pesquisa de campo, os hospitais universitários são vistos como fontes externas de aprendizado. No entanto, como se pode observar na Tabela 5, há, antes deles, outros agentes citados pelas empresas com maior grau de importância enquanto fontes de informação para inovação, como as "instituições de testes, ensaios e certificações”, os “órgãos públicos, inclusive de regulação" demonstrando a importância da ANVISA para produtos para a saúde, os próprios "clientes/beneficiários” e a parte de “distribuição, representantes e comércio”.

Tabela 5 - Fontes Externas de Aprendizado

\begin{tabular}{l|r|r|r|r|r}
\hline \multirow{2}{*}{ Fontes Externas } & \multicolumn{4}{c}{ Grau de Importância (\%) } \\
\cline { 2 - 6 } & Índice & Nula & Baixa & Média & Alta \\
\hline Instituições de testes, ensaios e certificações & $\mathbf{0 , 7}$ & 20,7 & 17,2 & 3,4 & 58,6 \\
Órgãos públicos, inclusive de regulação & $\mathbf{0 , 7}$ & 20,7 & 10,3 & 13,8 & 55,2 \\
Clientes / beneficiários & $\mathbf{0 , 7}$ & 6,9 & 13,8 & 27,6 & 51,7 \\
Distribuição, representantes e comércio & $\mathbf{0 , 7}$ & 10,3 & 13,8 & 34,5 & 41,4 \\
Universidades, inclusive hospitais universitários & $\mathbf{0 , 6}$ & 34,5 & 6,9 & 10,3 & 48,3 \\
Respectivos fornecedores & $\mathbf{0 , 6}$ & 0,0 & 34,5 & 27,6 & 37,9 \\
Serviços auxiliares / serviços técnicos & $\mathbf{0 , 6}$ & 10,3 & 24,1 & 31,0 & 34,5 \\
Associações e outras organizações representação e coordenação & $\mathbf{0 , 5}$ & 24,1 & 24,1 & 10,3 & 41,4 \\
\hline
\end{tabular}

Fonte: Pesquisa de campo (2012).

Nota: Índice $=\left(0^{*} n^{\circ}\right.$ nulas $+0,3 * n^{\circ}$ baixas $+0,6 * n^{0}$ médias $+n^{\circ}$ altas $) /\left(N^{\circ}\right.$ empresas). Quanto mais próximo de 1 for o resultado, maior o grau de importância da variável.

Com relação à localização dessas fontes externas, como é possível observar na Tabela 6, no geral, as fontes localizam-se na RMPA, no estado e no país. Universidades, inclusive hospitais universitários, consideradas como importantes fontes externas de conhecimento localizam-se especialmente na RMPA, justo onde se encontram os hospitais em análise.

Tabela 6 - Localização das Fontes Externas de Aprendizado

\begin{tabular}{|c|c|c|c|c|}
\hline \multirow{2}{*}{ Fontes Externas } & \multicolumn{4}{|c|}{ Localização (\%) } \\
\hline & RMPA & Estado & País & Exterior \\
\hline Instituições de testes, ensaios e certificações & 55,2 & 13,8 & 51,7 & 10,3 \\
\hline Órgãos públicos, inclusive de regulação & 51,7 & 13,8 & 58,6 & 0,0 \\
\hline Clientes / beneficiários & 79,3 & 69,0 & 75,9 & 6,9 \\
\hline Distribuição, representantes e comércio & 55,2 & 41,4 & 58,6 & 3,4 \\
\hline Universidades, inclusive hospitais universitários & 62,1 & 27,6 & 27,6 & 0,0 \\
\hline Respectivos fornecedores & 41,4 & 27,6 & 65,5 & 31,0 \\
\hline Serviços auxiliares / serviços técnicos & 79,3 & 13,8 & 13,8 & 0,0 \\
\hline Associações e outras organizações de representação e coordenação & 51,7 & 27,6 & 27,6 & 0,0 \\
\hline
\end{tabular}

Fonte: Pesquisa de campo (2012). Nota: Índice (ver nota na Tabela 5)

As empresas ainda foram questionadas sobre suas atividades cooperativas nos anos de 2009 a 2011. A identificação dos parceiros consta da Tabela 7. 
Tabela 7 - Parceiros em Atividades Cooperativas (2009-2011)

\begin{tabular}{l|r|r|r|r|r}
\hline \multirow{2}{*}{ Parceiros } & \multicolumn{4}{c}{ Grau de Importância (\%) } \\
\cline { 2 - 5 } & Índice & Nula & \multicolumn{1}{c}{ Baixa } & Média & Alta \\
\hline Organizações de apoio e promoção & $\mathbf{0 , 5}$ & 0,0 & 0,0 & 6,9 & 41,4 \\
Universidades, inclusive hospitais universitários & $\mathbf{0 , 4}$ & 0,0 & 0,0 & 6,9 & 31,0 \\
Fornecedores & $\mathbf{0 , 4}$ & 0,0 & 3,4 & 20,7 & 24,1 \\
Associações e outras organizações de representação e coordenação & $\mathbf{0 , 2}$ & 0,0 & 0,0 & 0,0 & 24,1 \\
Centros de capacitação profissional, de assistência técnica e de manutenção & $\mathbf{0 , 2}$ & 0,0 & 0,0 & 3,4 & 17,2 \\
Órgãos públicos, inclusive de regulação & $\mathbf{0 , 1}$ & 0,0 & 0,0 & 0,0 & 10,3 \\
Distribuição, representantes, comércio & $\mathbf{0 , 1}$ & 0,0 & 0,0 & 0,0 & 6,9 \\
Clientes / beneficiários & $\mathbf{0 , 1}$ & 0,0 & 0,0 & 0,0 & 6,9 \\
Instituto de pesquisa & $\mathbf{0 , 1}$ & 0,0 & 0,0 & 0,0 & 6,9 \\
Agentes financeiros & $\mathbf{0 , 1}$ & 0,0 & 0,0 & 0,0 & 6,9 \\
\hline
\end{tabular}

Fonte: Pesquisa de campo (2012).

Nota: Índice (ver nota na Tabela 5)

Os principais parceiros das empresas para realização de atividades cooperativas são as “organizações de apoio e promoção" com índice de 0,5 e as "universidades, inclusive hospitais universitários" e os "fornecedores”, ambos com índice de 0,4. Chama a atenção quão baixos são esses índices, o que demonstra a pouca relevância atribuída às parcerias pelas empresas da amostra.

Vale refletir sobre essa baixa interação entre as empresas e outros agentes, justo nesses segmentos industriais relacionados à saúde nos quais pressupõe-se a necessidade de conhecimentos científicos para a geração de inovações. A resposta para essa realidade está, em parte, dada pela característica dos produtos elaborados pela maioria das firmas, que pouco incorporam inovações tecnológicas.

Com relação à localização, as parcerias, quando ocorrem, se dão, sobretudo, com instituições localizadas predominantemente na RMPA, demonstrando a importância da proximidade geográfica para atividades de cooperação interorganizacional no caso das empresas examinadas.

Feitas essas considerações sobre as interações entre os vários subsistemas - de serviços, de ensino e pesquisa, e industrial -, verifica-se que essas são ainda tênues no sistema de saúde ora em análise. Cabe remarcar, no entanto, que as articulações entre as atividades científicas e as de assistência à saúde são mais visíveis do que aquelas entre as atividades científicas e a base produtiva regional, que são ainda pouco usuais. Assim, embora reconheça-se a presença e a densidade no sistema local de saúde de uma série de organizações vinculadas aos seus vários subsistemas, isso ainda não se reflete em um círculo virtuoso entre a geração de conhecimentos, a prestação de serviços hospitalares e as bases produtivas locais.

\section{Considerações Finais}

Em termos de interações entre grupos de pesquisa e organizações, identificou-se um número crescente na área de Ciências da Saúde no RS. Porém, releva destacar que apesar do crescimento nos últimos anos, o número total de interações ainda é muito baixo e insuficiente para desencadear processos virtuosos de aprendizagem e geração de inovações.

Do ponto de vista das interações entre as empresas industriais e outros agentes, verificou-se também uma fragilidade nas interações. Isso embora as características do setor saúde implicarem supor uma dependência de conhecimentos científicos para a geração de inovações. A resposta a esta realidade é, em parte, determinada pela característica dos produtos criados pela maioria das empresas analisadas, que são de baixa complexidade. Também é importante observar o tamanho das empresas da amostra; a maioria delas são micro e pequenas empresas. 
De toda forma, conforme demonstrou a pesquisa de campo, há uma grande oportunidade para que se avance na aproximação entre universidades e empresas. Isso não só na área das ciências da saúde, biológicas e afins, mas também no campo das engenharias. Conhecimentos dessas áreas exatas podem incrementar o progresso tecnológico das indústrias de base física, mecânica, eletrônica e de materiais voltadas à saúde. Da mesma forma que a tradição da indústria de base mecânica no RS traz oportunidades às empresas de instrumentação médica, que podem, por exemplo, obter ganhos de escala e ampliar seu poder de negociação junto a fornecedores através de parcerias com outros segmentos, como o de cutelaria.

Além disso, verificou-se que os hospitais desempenham um papel importante neste sistema. Mesmo que as interações existentes entre eles e os grupos de pesquisa / empresas são bastante tênues, entende-se que esses prestadores de serviços podem ser um importante espaço de inovação, especialmente na obtenção de impactos positivos relativos aos cuidados de saúde.

\section{Bibliografia}

ALBUQUERQUE, E. da M.; CASSIOLATO, J. E. As Especificidades do Sistema de Inovação do Setor Saúde. Revista de Economia Política, v. 22, n. 4 (88), outubro-dezembro 2002.

BARBOSA, P. R; GADELHA, C. A. G. O papel dos hospitais na dinâmica de inovação em saúde. Revista de Saúde Pública 46 (Supl), p. 68-75, 2012.

ASHEIM, B.; GERTLER, M. S. The geography of innovation: regional innovation systems. The Oxford Handbook of Innovation, Oxford University Press, Oxford, 2006.

BOTELHO, M. DOS R. A.; TATSCH, A. L. Health services and innovation in Brazil: an analysis based on teaching and research hospitals in Rio Grande do Sul and Minas Gerais. In: CASSIOLATO, J. E.; SOARES, M. C. C. (Eds.). Health systems, equity and development. Rio de Janeiro: E-papers, 2015. p. 355-381.

BRESCHI, S.; MALERBA, F. Sectoral Innovation Systems: Technological Regimes, Schumpeterian Dynamics, and Spatial Boundaries. In: EDQUIST, C. (Ed.). Systems of Innovation: technologies, institutions and organizations. London: Pinter, p.130-56, 1997.

CASSIOLATO, J. E.; SOARES, M. C. C. (Eds.). Health systems, equity and development. Rio de Janeiro: E-papers, 2015.

CHAVES, C. V.; ALBUQUERQUE, E. M. Desconexão no sistema de inovação do setor saúde: uma avaliação preliminar do caso brasileiro a partir de estatísticas de patentes e artigos. Revista de Economia Aplicada, v. 10, p. 523-539, 2006.

CHAVES, C. V.; MORO, S. Investigating the interaction and mutual dependence between science and technology. Research Policy, v. 36, p. 1204-1220, 2007.

COOKE, Philip. Introduction: origins of the concept. In: BRACZYK, Hans-Joachim; COOKE, Philip; HEIDENREICH, Martin (Ed.). Regional Innovation Systems. London: UCL Press, 1998. p 2-25.

COSTA, A. B. da; RUFFONI, J.; PUFFAL, D. Proximidade Geográfica e Interação UniversidadeEmpresa no Rio Grande do Sul. Revista de Economia (Curitiba), v. 37, p. 213- 238, 2011.

DJELLAL, F.; GALLOUJ, F. Mapping innovation dynamics in hospitals. Research Policy, 34, p. 817835, 2005.

EDQUIST, C. (Ed.). Systems of innovation: technologies, institutions, and organizations. Londres: Pinter, 1997.

FREEMAN, C. The 'National System of Innovation' in Historical Perspective. Cambridge Journal of Economics, 19, p. 5-24, 1995.

FUNDAÇÃO OSWALDO CRUZ et al. A saúde no Brasil em 2030: diretrizes para a prospecção 
estratégica do sistema de saúde brasileiro. Rio de Janeiro: Fiocruz/Ipea/ Ministério da Saúde/Secretaria de Assuntos Estratégicos da Presidência da República, 2012.

GADELHA, C. A. G. O complexo industrial da saúde e a necessidade de um enfoque dinâmico na economia da saúde. Ciência \& Saúde Coletiva, v. 8, n. 2, p. 521-35, 2003.

GADELHA, C. (Coord.). Perspectivas do investimento em saúde. Rio de Janeiro: UFRJ, 2008 / 2009. Relatório final do estudo do sistema produtivo Saúde, integrante da pesquisa "Perspectivas do Investimento no Brasil”, realizada por IE/UFRJ e IE/UNICAMP. Disponível em: http://www.projetopib.org/?p=documentos

GADELHA, C. A. G. (Coord.). A dinâmica do sistema produtivo da saúde: inovação e complexo econômico-industrial. Rio de Janeiro: Editora FIOCRUZ, 2012.

GELIJNS, A. C.; ROSEMBERG, N. The changing nature of medical technology development. In: ROSEMBERG, N.; GELIJNS, A. C.; DAWKINS, H. Sources of medical technology: universities and industry. Washington: National Academy Press, 1995.

HICKS D.; KATZ, J. S. Hospitals: the hidden research system. Science and Public Policy 23(5), 297304, 1996.

INSTITUTO BRASILEIRO DE GEOGRAFIA E ESTATÍSTICA - IBGE. Pesquisa de Inovação 2011. Rio de Janeiro: IBGE, 2013.

JOHNSON, B. Introduction of Part I Systems of innovation: overview and basis concepts. In: EDQUIST, C. (Ed.). Systems of innovation: technologies, institutions, and organizations. Londres, 1997.

LAMBERTY, M. J. O sistema de inovação em saúde: um estudo sobre as empresas industriais de equipamentos médicos, hospitalares e odontológicos gaúchas. São Leopoldo: Unisinos, 2014. Dissertação (Mestrado em Economia), Pós-Graduação em Economia, Universidade do Vale do Rio dos Sinos, 2014.

LUNDVALL, B-Å. (Ed.). National innovation systems: towards a theory of innovation andinteractive learning. London: Pinter, 1992.

LUNDVALL, B- $\AA$ et al. National systems of production, innovation and competence building. Research Policy, n. 31, p. 213-231, 2002.

NELSON, R. (Ed.). National Innovation Systems: a comparative analysis. Nova York: Oxford University, 1993.

NELSON, R. R.; BUTERBAUGHB, K.; PERLB, M.; GELIJNS, A. How medical know-how progresses. Research Policy, n. 40, p. 1339-1344, 2011.

STEFANI, R. Inovação da firma e dinâmicas de proximidade: o caso da indústria de equipamentos medicos, hospitalares e odontológicos do RS. São Leopoldo: Unisinos, 2015. Dissertação (Mestrado em Economia), Pós-Graduação em Economia, Universidade do Vale do Rio dos Sinos, 2015.

TATSCH, A. L.; BATISTI, V.; FRAGA, W. S. O sistema inovativo da saúde gaúcho: uma análise a partir do caso de Porto Alegre voltado aos tratamentos cardiovasculares e oncológicos. In: Anais do XI Encontro Nacional da Associação Brasileira de Estudos Regionais e Urbanos - XI ENABER. Foz do Iguaçu: Enaber, 2013.

WINDRUM, P.; GARCÍA-GOÑI, M. A neo-schumpeterian model of health services innovation. Research Policy, 37, p. 649-672, 2008. 